\title{
Prognostic significance of DNA cytometry in carcinoma of the uterine cervix FIGO stage IB and II
}

\author{
Hans Jürgen Grote ${ }^{a}$, Nicolaus Friedrichs ${ }^{b}$, \\ Natalia Pomjanski a , Helen Friderike Guhde ${ }^{\text {a }}$, \\ Olaf Reich ${ }^{\mathrm{c}}$ and Alfred Böcking ${ }^{\mathrm{a}, *}$ \\ ${ }^{a}$ Institute of Cytopathology, \\ Heinrich-Heine-University, Moorenstrasse 5, \\ D-40225 Düsseldorf, Germany \\ ${ }^{\mathrm{b}}$ Institute of Pathology, \\ Friedrich-Wilhelms-University, Regina-Pacis-Weg 3, \\ D-53113 Bonn, Germany \\ ${ }^{\mathrm{c}}$ Department of Obstetrics and Gynecology, \\ Karl-Franzens-University, Universitätsplatz 3, \\ A-8010 Graz, Austria
}

Received November 2001

Accepted 18 November 2001

Objective: To assess the prognostic value of DNA-image cytometry in cervical carcinoma of the uterus and its relation to other established prognostic factors.

Study design: The study included 116 cases of cervical carcinoma FIGO stages IB and II which were treated with radical abdominal hysterectomy. The median follow-up was 55 months (range 1-162 months). DNA image cytometry was performed on cytologic specimens prepared by enzymatic cell separation from formalin-fixed, paraffin-embedded tissues. DNA stemline ploidy, DNA stemline aneuploidy, 5c exceeding rate, $9 \mathrm{c}$ exceeding rate, $2 \mathrm{c}$ deviation index, and DNA malignancy grade were computed. DNA-variables as well as various clinical and histological variables were related to survival rates.

Results: In multivariate statistical analysis DNA stemline ploidy using $2.2 \mathrm{c}$ as a cut-off value and FIGO stage showed to be statistically significant available presurgery predictors of survival, whereas the postsurgical parameters lymphonodal status, tumor size and parametrial involvement were significantly correlated with survival. The synopsis of all pa-

\footnotetext{
${ }^{*}$ Corresponding author: Prof. Dr. Alfred Böcking, Institute of Cytopathology, Heinrich-Heine-University, Moorenstrasse 5, D-40225 Düsseldorf, Germany, Tel.: +49-211 81 18346; Fax: +49 21181 18402; E-mail: boecking@uni-duesseldorf.de.
}

rameters in a multivariate Cox model indicated that - with declining relevance - the number of positive pelvic lymph nodes, DNA stemline ploidy using a cut-off level at a modal value of $2.2 \mathrm{c}$, largest pelvic lymph node, $5 \mathrm{c}$ exceeding rate, and ratio of carcinoma area to cervix area, were of predictive value for survival.

Conclusions: Our results suggest that prognostic information deducted from classical staging parameters is successfully complemented by DNA image cytometry which can be applied pretherapeutically.

Keywords: Cervical cancer, staging, DNA image cytometry, aneuploidy, prognosis, grading

\section{Introduction}

Accurate prediction of the biologic behavior of malignant neoplasms is important in helping to determine patient management and in designing therapeutic trials. Clinical staging of cervical carcinomas so far, has been the most important single parameter influencing choice of treatment and indicating outcome [4]. However, it has some shortcomings: clinical staging has failed to predict the surgical stage correctly in a significant number of cases. Discrepancies in at least $1 / 3$ of cases have been reported [26,28]. In addition, clinical staging allows significant variation in tumor volume within stages. Tumor volume of cervical carcinoma has been proposed to be even more important, both in early stage and in locally advanced stage [8].

The cytophotometric identification of DNA distribution abnormalities has been shown to be prognostically important in several human malignancies including, e.g., prostate, bladder, and breast cancer $[1,3,34]$. In squamous cell carcinoma of the cervix, the results have been conflicting. Some studies have found a prognostic impact of DNA ploidy and/or S-phase fraction [21,24, $33,35]$, while others were not able to confirm this [23, 37]. Most of these studies have been performed using DNA flow cytometry. Studies which applied DNA im- 
age cytometry agreed concerning the prognostic value of DNA cytometry, but these studies originated mostly in the 1970s and 1980s [2,9,15]. Meanwhile major effort has been undertaken to standardize DNA image cytometry $[6,14,16,17]$.

The aim of the present study was to evaluate the prognostic significance of standardized DNA-imagecytometry on biopsies of cervical carcinoma in comparison to several clinical and histological variables.

\section{Material and methods}

\subsection{Patients}

Among the patients with cervical cancer who were treated in the Department of Obstetrics and Gynecology, University of Graz, Austria, from January 1977 to December 1988,116 patients with primary invasive cervical carcinoma in FIGO Stages IB, IIA, and IIB were eligible for this study because they met the following conditions: a biopsy containing tumor tissue was available before surgery, the primary treatment was radical abdominal hysterectomy with pelvic lymph node dissection, postsurgical staging and a complete clinical follow-up were documented, and survival status to the date of last follow-up (on June 30, 1991) was known. The mean age of the patients was $47.2 \pm 10.4$ years (SD). The median follow-up time was 55 months (range 1-162 months). Among 54 patients with recurrent disease, 43 died and 11 were still alive with tumors for which they were receiving palliative therapy at the closing date of the study. Five patients intercurrently died of a disease unrelated to cervical cancer. Postsurgical adjuvant treatment was not allowed for the estimation of survival rates.

\subsection{Pathology}

All biopsy specimens were recut from the paraffinembedded tissue blocks for hematoxylin-eosin (H\&E) staining and reexamined by one of the authors (A.B.). Histologic type and grade was redetermined according to the World Health Organization classification [32]. Mitotic rate, lymphovascular space involvement, carcinoma area, cervix area, ratio of carcinoma area to cervix area, microscopic evidence of parametrial involvement, number of dissected parametrial lymph nodes, number of pelvic lymph node metastases, and size of largest pelvic lymph node were retrieved from the original pathology reports.

\subsection{Image cytometry}

Those areas of the H\&E-stained sections of the biopsy specimens which were relevant for the histologic diagnosis were marked in order to prepare tumor cell monolayer smears by a cell separation technique described elsewhere in detail [6]. In brief, from the area of the tissue block corresponding to the marked area of the H\&E-stained section, three $50 \mu$ m-thick sections were cut and placed in a glass tube. The material was deparaffinized with xylene, rehydrated and washed in a phosphate-buffered solution (PBS) (pH 7.2). Afterwards it was suspended in $0.5 \%$ pepsin in $0.2 \% \mathrm{HCl}$ and incubated at $37^{\circ} \mathrm{C}$ for 30 minutes. The pepsinized material was then centrifuged and mechanically dispersed with an Ultra-Turrax homogenizer (Janke \& Kunkel, Staufen, Germany) using 10,000 rpm for 30 seconds. After mechanical separation the suspension was filtered through a $70 \mu \mathrm{m}$ nylon mesh, centrifuged and smeared on poly-L-lysine-coated slides.

Feulgen staining was performed automatically in a modified staining machine, Varistain-24 (Shandon, Pittsburgh, Pennsylvania, USA), as described previously [11]. In brief, acid hydrolysis $\left(4 \mathrm{~N} \mathrm{HCl}, 27.5^{\circ} \mathrm{C}\right.$, 55 minutes) was followed by 60 -minute incubation in Schiff's reagent (Merck, Darmstadt, Germany) at room temperature.

Measurements were performed with a TV image analysis system, the MIAMED-DNA (Leitz, Wetzlar, Germany) combined with an automated microscope. Details of the measurement procedure were described by Sanchez et al. [31]. The configuration included a Saticon 1" TV-camera (Bosch, Stuttgart, Germany), a stabilized power supply for a $12 \mathrm{~V}$ halogen lamp, and a $570 \mathrm{~nm}$-interference filter with a halfwidth of $10 \mathrm{~nm}$. Measurements were performed using a 40/0.70 objective. In each case 200 tumor cells were measured at random. At least 20 lymphocytes were measured as reference cells for DNA parameters; the mean of their integrated optical density was multiplied by a correction factor of 1.25 to obtain the normal $2 \mathrm{c}$ value. The coefficient of variation $(\mathrm{CV})$ of the reference cell populations was below $5 \%$.

The following parameters were calculated:

- DNA stemline ploidy:

DNA stemline ploidy was defined as the modal value of a DNA stemline [17]. Two subgroups of DNA stemline ploidy were differentiated using a cut-off level at a modal value of 2.2c. 
- DNA stemline aneuploidy:

DNA stemline aneuploidy was assumed, if the modal value of a DNA stemline was $<1.80 \mathrm{c}$ and $>2.20 \mathrm{c}$ or $<3.60 \mathrm{c}$ and $>4.40 \mathrm{c}[16]$.

- The $5 \mathrm{c}$ exceeding rate $(5 \mathrm{cER})$ and the $9 \mathrm{c}$ exceeding rate $(9 \mathrm{cER})$ :

are defined as the percentage of aneuploid cells having a DNA content of more than $5 \mathrm{c}$ or $9 \mathrm{c}$, respectively [5]; DNA aneuploidy was detected by a single-cell interpretion if at least three $5 \mathrm{c}$ exceeding events $(5 \mathrm{cEE})$ and/or at least three 9cexceeding events (9cEE) [10] were found.

- The $2 \mathrm{c}$ deviation index (2cDI):

defined as the mean square deviation of $n$ measured tumor cells, $c_{i}$, from the diploid value representing the variance of the tumor cell population around the normal $2 \mathrm{c}$ value [16]:

$$
2 \mathrm{cDI}=\frac{1}{n} \times \sum_{i=1}^{n}\left(c_{i}-2 \mathrm{c}\right)^{2} .
$$

- The DNA malignancy grade: was calculated from the $2 \mathrm{cDI}$ value as previously described [5].

\subsection{Statistical analysis}

Overall survival time was calculated from the date of surgery to the date of last follow-up or death caused or accompanied by clinical evidence of cervical carcinoma. Patients dying from causes unrelated to cervical cancer were censored at the time of death. The Kaplan-Meier method was used to estimate the survival rate and survival curve in each parameter, and the differences between curves were assessed using the Wilcoxon-Breslow test. Statistical significance of univariate analysis was defined as $P<0.05$. Cox's proportional hazards model was used to evaluate the prognostic power of various factors in multivariate analysis. All of the statistical calculations were done using BMDP software package (Department of Biomathematics, University of California, Los Angeles, USA) [12]. This software package used $P>0.1$ as a cut-off value for the exclusion of a variable from the Cox model.

\section{Results}

The DNA cytometric, clinical, and histological parameters which were significantly correlated with sur- vival are summarized in the first column of Table 1. The availability of these prognostic factors either before or after surgery is indicated. For statistical analysis 116 patients were stratified by FIGO stage. When the strata differed with respect to the characteristic under study they were placed on top of each other, otherwise next to each other (column 2). Subgroups in which the feature under study did not show a significant statistical correlation with survival were for the most part omitted from this table to simplify the presentation. The number of patients within the subgroups is given in column 3. The prognostic implication of a characteristic is listed in the fourth column. Finally, the last column shows the $p$-value of the respective prognostic statement. If necessary, the strata in which the prognostic value of a particular parameter was tested and the respective $p$-values are marked, e.g., with an asterisk.

Presurgically 46 of the 116 patients were assigned to FIGO stage IB and 70 to stage II. All of the latter presented with a stage IIB-tumor with exception of one patient who revealed a stage IIA tumor. Out of 46 patients belonging to the FIGO stage IB-subgroup $13(28.3 \%)$ had a recurrent disease and 11 of these (24.0\%) died of cervical cancer. One died of a disease unrelated to her cervical carcinoma. In contrast $41(58.6 \%)$ out of 70 patients with a FIGO stage IItumor had a recurrent disease and 29 of these $(41.4 \%)$ died of their cervical cancer. Within this subgroup an additional four patients died of unrelated disease. The different survival rates are illustrated in Fig. 1 by the Kaplan-Meier method and were statistically significant in univariate analysis $(p=0.01)$. The age of the patients did not correlate with the clinical outcome.

Like FIGO staging, the results of DNA image cytometry are available before surgery when the measurements are performed on biopsy specimens after enzymatic cell separation. In this study tumors with a DNA stemline ploidy $\leqslant 2$. 2 c showed a significantly better survival than hyperdiploid tumors (Fig. 2, $p<0.05)$. The prognostic implication was valid both for patients with stage IB- and stage II-tumors. Four cases were excluded from the statistical analysis, because it was not possible to define a DNA stemline in the DNA histogram. The stratification into three DNAploidy-groups $(<1.80 \mathrm{c}$ and $>2.20 \mathrm{c}$ or $<3.60 \mathrm{c}$ and $>4.40$ c) according to Haroske et al. [16] did not discriminate statistically significant prognosis (data not shown). Nevertheless there was an obvious tendency for a better survival of patients with diploid tumors as compared to those with hyperdiploid tumors which might have become statistically significant in a larger 
Table 1

Clinicopathologic and DNA image cytometric characteristics and their relation to survival (univariate analysis)

\begin{tabular}{|c|c|c|c|c|}
\hline Characteristic & FIGO stage $^{\mathrm{a}}$ & $n$ & Prognosis & $P$ \\
\hline \multicolumn{5}{|c|}{ Presurgery } \\
\hline \multicolumn{5}{|l|}{ FIGO stage } \\
\hline $\mathrm{Ib}$ & $\mathrm{Ib}$ & 46 & favorable & 0.01 \\
\hline II & II & 70 & unfavorable & \\
\hline \multicolumn{5}{|l|}{ DNA stemline ploidy } \\
\hline$\leqslant 2.20$ & $\mathrm{Ib}, \mathrm{II}$ & 24 & favorable & $<0.05$ \\
\hline$>2.20$ & $\mathrm{Ib}, \mathrm{II}$ & 88 & unfavorable & \\
\hline \multicolumn{5}{|c|}{ Postsurgery } \\
\hline Carcinoma area $\leqslant 349 \mathrm{~mm}^{2}$ & $\mathrm{Ib}$ & 23 & favorable & $<0.01$ \\
\hline Carcinoma area/cervix area $\leqslant 0.36$ & $\mathrm{Ib}$ & 23 & favorable & $<0.05$ \\
\hline \multicolumn{5}{|l|}{ Lymphvessel invasion } \\
\hline No & $\mathrm{Ib}$ & 22 & favorable & $<0.05$ \\
\hline Yes & $\mathrm{Ib}$ & 24 & unfavorable & \\
\hline \multicolumn{5}{|l|}{ Histologic parametrial involvement } \\
\hline \multirow[t]{2}{*}{ No } & $\mathrm{Ib}^{*}$ & 34 & favorable & $<0.001^{\mathrm{b}}$ \\
\hline & II & 43 & & \\
\hline \multirow[t]{2}{*}{ Yes } & $\mathrm{Ib}^{* *}$ & 12 & unfavorable & \\
\hline & II & 27 & & \\
\hline \multicolumn{5}{|l|}{ No. of parametrial lymph nodes } \\
\hline$\leqslant 2$ & $\mathrm{Ib}, \mathrm{II}$ & 45 & & \\
\hline $3-5^{\dagger}$ & $\mathrm{Ib}, \mathrm{II}$ & 44 & & \\
\hline$>5^{\ddagger}$ & $\mathrm{Ib}, \mathrm{II}$ & 27 & unfavorable & $<0.01^{\mathrm{b}}$ \\
\hline \multicolumn{5}{|l|}{ Pelvic lymph nodes } \\
\hline \multirow[t]{2}{*}{ Negative } & $\mathrm{Ib}^{\#}$ & 25 & favorable & $<0.05^{\mathrm{b}}$ \\
\hline & $\mathrm{II}^{\dagger}$ & 30 & & $0.01^{\mathrm{b}}$ \\
\hline \multirow[t]{2}{*}{ Positive } & $\mathrm{Ib}^{\# \#}$ & 21 & unfavorable & \\
\hline & $\mathrm{II}^{\ddagger}$ & 40 & & \\
\hline \multicolumn{5}{|l|}{ No. of positive pelvic lymph nodes } \\
\hline $0^{\#}$ & $\mathrm{Ib}, \mathrm{II}$ & 55 & favorable & $<0.05^{\mathrm{b}}$ \\
\hline$\leqslant 2^{\# \#}$ & $\mathrm{Ib}, \mathrm{II}$ & 35 & unfavorable & $0.01^{\mathrm{b}}$ \\
\hline$>2^{\ddagger \ddagger}$ & $\mathrm{Ib}, \mathrm{II}$ & 26 & unfavorable & \\
\hline \multicolumn{5}{|l|}{ Largest positive pelvic lymph node } \\
\hline$\leqslant 10 \mathrm{~mm}^{2}$ & II & 16 & unfavorable & $<0.05$ \\
\hline$>10 \mathrm{~mm}^{2}$ & II & 23 & & \\
\hline
\end{tabular}

study group (data not shown). The DNA proliferation fraction, the $5 c$ and $9 \mathrm{c}$ exceeding rates, the $2 \mathrm{c}$ deviation index, and the DNA malignancy grade did not prove as valid predictors of survival in univariate analysis.

The histologic type of the tumors was predominantly squamous with 111 squamous cell carcinomas, four adenocarcinomas, and one adenosquamous carcinoma. The histologic type failed to show any statistically significant relation to survival. But there was a tendency of unfavorable clinical outcome in cases with small cell non keratinizing squamous cell carcinoma which might have been statistically significant in a larger study group. No correlation with clinical outcome was found for the mitotic rate.

Postsurgical histological parameters available were retrieved from the original pathology reports. Among them, tumor size turned out to be a prognostically significant parameter only in patients without clinically diagnosed parametrial involvement (FIGO stage IB). In this study small tumors with a carcinoma area 


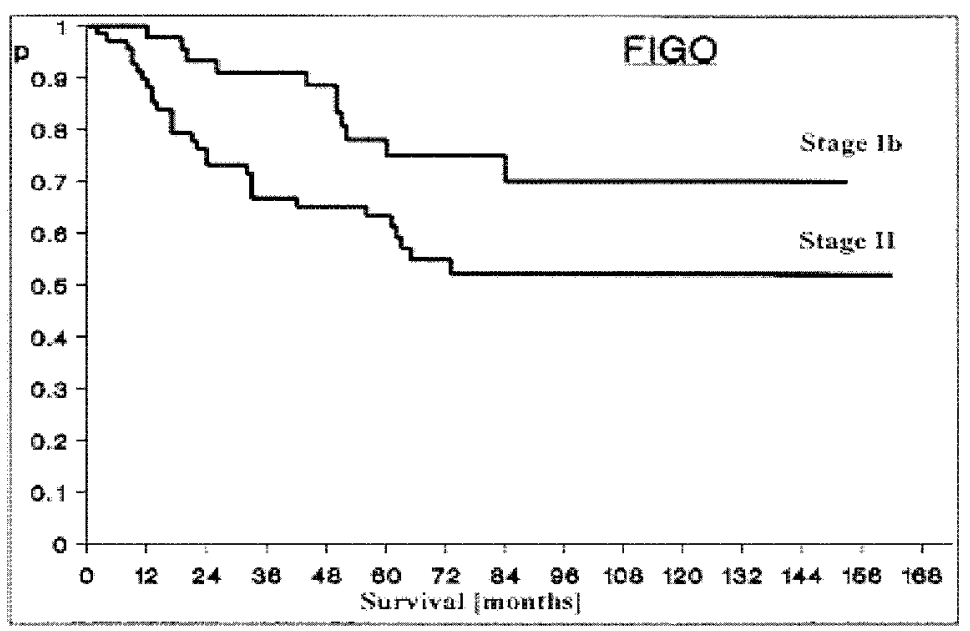

Fig. 1. Time of survival (months) correlated with clinical stage according to FIGO. The two groups constitute 46 (stage IB) and 70 (stage II) patients, respectively. $P=0.01$.

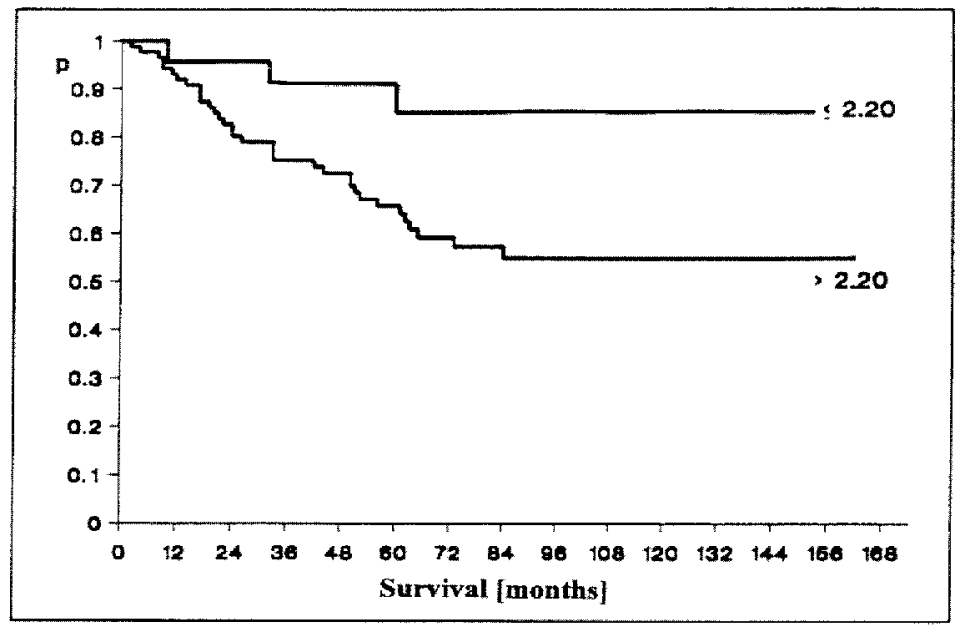

Fig. 2. Survival in months according to DNA ploidy using $2.2 \mathrm{c}$ as a cut-off value $(P<0.05)$. The two groups constitute $24(\leqslant 2.2 \mathrm{c})$ and 88 ( $>2.2 \mathrm{c})$ patients, respectively.

$\leqslant 349 \mathrm{~mm}^{2}(p<0.01)$ or a carcinoma area/cervix area-ratio $\leqslant 0.36(p<0.05)$ were correlated with a favorable clinical outcome on condition that the tumor belongs to FIGO stage IB. Tumor size was no longer prognostically relevant in patients with a stage II-tumor (data not shown).

Like tumor size, lymphvessel invasion proved to be a prognostically valid parameter only in patients without clinically diagnosed parametrial involvement (FIGO stage IB). In these patients evidence of lymphvessel invasion was an indicator of poor clinical outcome $(p<0.05)$.

Parametrial involvement is a parameter which enters into the FIGO-staging system. Table 1 shows that the correlation of clinical and histological evaluation of parametrial involvement is poor. In only 61 out of 116 patients $(52.6 \%)$ the clinical assessment of parametrial involvement performed presurgically was in accordance with postsurgical histopathologic examination. Patients who were assigned to FIGO stage IB and turned out to have histologic parametrial involvement showed an unfavorable prognosis $(p<0.001)$. Interestingly enough, the lacking histologic proof of parametrial involvement did not influence the survival of patients with a tumor clinically estimated at stage II.

Both patients with stage IB- and stage II-tumors showed an unfavorable prognosis if a high number $(>5)$ of parametrial lymph nodes was dissected 
$(p<0.01)$. There was no correlation between clinical outcome and dissection of 5 or less parametrial lymph nodes. The presence of carcinomatous involvement of the dissected parametrial lymph nodes was not investigated in this study.

The evidence of pelvic lymph node metastases and its number was of prognostic significance both for stage IB- and II-tumors. The results of this study show that tumorous lymph nodes are a frequent finding in both stages and that this is followed by a deteriorated clinical outcome (stage IB: $p<0.05$; stage II: $p<0.01)$. Twenty-one $(45.7 \%)$ out of 46 tumors in stage IB and $40(57.1 \%)$ out of 70 tumors in stage II had pelvic lymph node involvement. In addition, the detection of more than two pelvic lymph node metastases resulted in an even more unfavorable prognosis $(p<0.01)$.

Finally, in stage II-tumors the size of the largest pelvic lymph node was relevant for the prognosis. Patients with a pelvic lymph node $>10 \mathrm{~mm}^{2}$ had an unfavorable clinical outcome as compared with those with a largest pelvic lymph node $\leqslant 10 \mathrm{~mm}^{2}(p<0.05)$.

Multiparameter analysis. Cox stepwise regression analysis of the presurgical parameters revealed FIGO stage, DNA stemline ploidy using $2.2 \mathrm{c}$ as a cut-off value, $5 \mathrm{cER}$, and mitotic rate as independent predictors of survival (Table 2). The multivariate analysis of postsurgery parameters available showed that the number of tumorous pelvic lymph nodes, the size of the biggest pelvic lymph node, and the ratio of carcinoma area to cervix area were prognostically significant (data not shown). Finally, Cox stepwise regression analysis of all investigated parameters revealed the number of positive pelvic lymph nodes, DNA stemline ploidy using a cut-off level at a modal value of $2.2 \mathrm{c}$, largest pelvic lymph node, $5 c$ exceeding rate, carcinoma area/cervix area-ratio, and DNA-proliferation fraction to be of independent prognostic significance with declining relevance (Table 3 ). In contrast FIGO stage did not retain independent prognostic significance after inclusion of the postsurgery available parameters into the Cox model.

For validation of the Cox model, the individual risk of the patients resulting from their individual profile of prognostically relevant factors and regression coefficients was calculated by a risk function: $R=\mathrm{EXP}$ $(1.1906 \mathrm{RCC}+0.1106 \mathrm{NLN}+0.0434 \mathrm{SLN}+0.7599$ $\mathrm{STL}+(-0.03435 \mathrm{cER}))$. The cutpoints of prognostically relevant factors used were 0.31 and 0.74 for RCC (ratio of carcinoma area to cervix area), 0 and 2 for NLN (number of pelvic lymph node metastases), $10 \mathrm{~mm}^{2}$ for SLN (size of the largest pelvic lymph node), 2.2c for STL (DNA stemline ploidy), and 7.05 and 22.05 for $5 \mathrm{cER}$ (5c exceeding rate). According to the quantile of the distribution three risk groups were formulated (RI: $n=28$; RII: $n=56$; RIII: $n=28$ ). The median survival time differed significantly between the three groups (RI vs. RII: $p<0.05$; RII vs. RIII: $p<0.001)$ and is documented in Fig. 3.

Table 2

Presurgery available prognostic factors in multivariate analysis (Cox proportional hazards regression model)

\begin{tabular}{lccccc}
\hline Variable & \multicolumn{2}{c}{ Improvement } & & \multicolumn{2}{c}{ Global } \\
\cline { 2 - 3 } \cline { 5 - 6 } & Chi-square & $P$ value & & Chi-square & $P$ value \\
\hline FIGO stage & 4.95 & 0.026 & & 4.71 & 0.030 \\
DNA stemline ploidy & 3.15 & 0.076 & & 7.68 & 0.022 \\
5cER & 7.02 & 0.008 & & 13.61 & 0.003 \\
Mitotic rate & 3.98 & 0.046 & & 18.06 & 0.001 \\
\hline
\end{tabular}

Table 3

Postsurgery synopsis of prognostic factors in multivariate analysis (Cox proportional hazards regression model)

\begin{tabular}{lccccr}
\hline Prognostic variable & \multicolumn{2}{c}{ Improvement } & & \multicolumn{2}{c}{ Global } \\
\cline { 2 - 3 } \cline { 5 - 6 } & Chi-square & $P$ value & & Chi-square & $P$ value \\
\hline No. of positive pelvic lymph nodes & 15.88 & 0.000 & & 25.11 & 0.000 \\
DNA stemline ploidy & 3.51 & 0.061 & 28.30 & 0.000 \\
Largest pelvic lymph node & 3.28 & 0.070 & 31.59 & 0.000 \\
5cER & 3.51 & 0.061 & 32.60 & 0.000 \\
Carcinoma area/cervix area & 3.21 & 0.073 & 34.62 & 0.000 \\
\hline
\end{tabular}




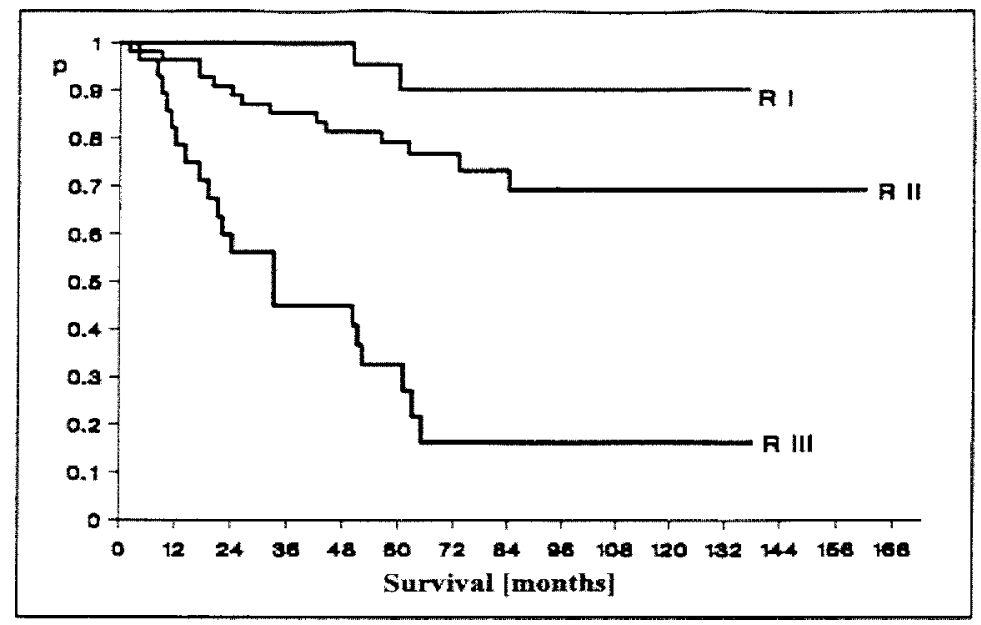

Fig. 3. Survival rates by risk groups. Significantly better survival was in the low risk RI-subgroup $(n=28)$ as compared to the intermediate risk RII-subgroup $(n=56)$, and in the intermediate risk RII-subgroup as compared to the high risk RIII-subgroup $(n=28)(P<0.05$ and $P<0.001$, respectively).

\section{Discussion}

Although there has been considerable interest in diagnostic DNA cytometry for an objective assessment of tumor prognosis, its utility for patients with cancer of the cervix is still in question. Jakobsen and coworkers [21] and Lai et al. [24] showed that aneuploid tumors with a high relative DNA content were associated with a poorer prognosis. The Scandinavian study [21] connected a DNA index above 1.5 with an unfavorable outcome. Lai et al. [24] suggested a DNA index of 1.3 as a cut-off value. In contrast to this, Atkin and colleagues [2] and Rutgers et al. [30] found that DNA peridiploid tumors were most aggressive. Two prospective studies concerning the prognostic information of DNA flow cytometry in cervical squamous cell carcinoma linked a high S-phase fraction (SPF) in near-diploid tumors with an unfavorable outcome [33, 35]. However, DNA ploidy level had no prognostic significance when SPF was not included in the multivariate analysis. Other authors have failed to find any correlation between DNA ploidy or SPF and survival [23, 37].

In this study, the prognostic value of several variables measured by DNA-image-cytometry was compared with comprehensive information about clinical and histopathological variables. All biopsy specimens analyzed originated from patients with cervical carcinoma treated with radical abdominal hysterectomy and bilateral pelvic lymphadenectomy. The results showed that a DNA stemline ploidy above $2.2 \mathrm{c}$ was significantly related to survival in univariate analysis and that it was the second most relevant prognostic factor both presurgically and postsurgically in multivariate analysis. Before surgery, its prognostic significance was only surpassed by clinical stage. After hysterectomy and pelvic lymph node dissection, solely the number of pelvic lymph node metastases achieved more important prognostic significance than DNA-stemline ploidy whereas FIGO stage lost its independent prognostic value in multivariate analysis.

DNA image cytometry and flow cytometry are objective methods to study abnormalities of nuclear DNA distribution. However, because of the different methods of preparation of nuclear suspensions and histogram interpretation, substantial subjectivity existed so far especially with regard to older studies. Lacking standardization may partially explain why the published rates of non-diploid tumors (e.g., Lai et al. [24], Jakobsen [20]) in cervical carcinoma vary widely (35$80 \%$ ) and is likely to have had a major impact on the prognostic validity of diagnostic DNA cytometry in the past. In the present study $78 \%$ of tumors revealed DNA-stemlines above the cut-off value of $2.2 \mathrm{c}$. In 1995 a European consensus report on standardization of diagnostic DNA image cytometry was presented [7] to overcome the problem of lacking standardization and has since then been updated twice $[14,16,17]$. To the best of our knowledge all data available in literature on the prognostic significance of DNA image cytometry in cervical squamous cell carcinoma were generated before 1995 [2,9,15,25].

The majority of investigations on prognostic value of DNA cytometric analysis have been performed by 
flow cytometry [21,23,24,30,33,35,37]. Still, disadvantages of flow cytometry are its inability to measure mixed populations separately along with the lacking visual control of cells under investigation [6]. In addition, flow cytometric studies frequently do not apply an internal or external standard. Many investigators assigned a diploid DNA amount to the histogram peak revealing lowest DNA content, even if it might have represented a hypo- or hyperdiploid DNA stemline. Consequently the lack of an internal reference may result in overlooking hypo- or hyperdiploid (peridiploid), but nevertheless aneuploid tumor cell populations.

Another possible explanation for the indecision regarding the usefulness of DNA cytometry in determinating the prognosis of patients with cervical cancer is that the choice of therapeutic regimen interferes with the survival of patients with aneuploid tumors and/or tumors with high SPF. Several authors suggested that DNA-aneuploid tumors may be more sensitive to radiation therapy $[13,25,36]$. The improved response rate in DNA-aneuploid tumors is likely to be related to the higher percentage of cells in the $\mathrm{S}+\mathrm{G} 2 / \mathrm{M}$ phases of the cell cycle, since the killing effect of radiation is cell-cycle dependent and aneuploid tumors often have a higher proliferative rate compared to diploid tumors. Atkin [2] and Rutgers et al. [30] who connected nondiploid tumors to a favorable prognosis exclusively investigated patients who underwent primary radiation therapy. On the other hand, Naus and Zimmerman [27], Willén et al. [35], and Kristensen et al. [23] investigated a heterogenous population in regard to the therapeutic regimen. They stated that their data did not demonstrate any differences between surgical or radiation therapy alone.

The $5 \mathrm{c}$ exceeding rate $(5 \mathrm{cER})$ is another variable that was found to be correlated with survival in multivariate analysis in this study. 5cER is defined as the percentage of cells exceeding a DNA content of $5 \mathrm{c}$ and has been used for the grading of malignancy [5]. The $9 c$ exceeding rate, $2 c$ deviation index, and DNA malignancy grade had no prognostic significance.

Beside the measurements by DNA image cytometry the present study compiled data on several clinical and histopathological variables. Like other authors we found clinical stage and number of pelvic lymph node metastases to be the most important prognostic factors presurgically and postsurgically, respectively $[4,19]$. However, the present study confirms the observation $[26,28]$ that presurgical clinical staging fails to predict parametrial involvement in a significant number of cases correctly. In only $54 \%$ of the cases FIGO stage was in accordance with postsurgical histopathologic examination of parametrial involvement (Table 1).

The prognostic significance of tumor size, ratio of carcinoma area to cervix area, lymph vessel invasion, mitotic rate, parametrial invasion, number of parametrial lymph nodes, and largest pelvic lymph node is in accordance with other studies [4,18,22]. Age, histologic type, and grade have been associated with survival, but a review of the literature shows contradictory view-points $[4,29]$. The present study did not reveal such statistically significant correlations.

In conclusion, the results of this study confirm the prognostic value of standardized DNA image cytometry and suggest that a DNA stemline ploidy above $2.2 \mathrm{c}$ is correlated with an unfavorable prognosis of cervical carcinoma. Therefore, the assessment of DNA stemline ploidy could lead to a better definition of subsets of patients requiring a distinctive treatment.

\section{References}

[1] H. al-Abadi and R. Nagel, Transitional cell carcinoma of the renal pelvis and ureter: prognostic relevance of nuclear deoxyribonucleic acid ploidy studied by slide cytometry: an 8-year survival time study, J. Urol. 148 (1992), 31-37.

[2] N.B. Atkin, Prognostic significance of ploidy level in human tumors: I. Carcinoma of the uterus, J. Natl. Cancer Inst. 56 (1976), 909-910.

[3] C.B. Bagwell, G.M. Clark, F. Spyratos, A. Chassevent, P.O. Bendahl, O. Stal, D. Killander, M.L. Jourdan, S. Romain, B. Hunsberger and B. Baldetorp, Optimizing flow cytometric DNA ploidy and S-phase fraction as independent prognostic markers for node-negative breast cancer specimens, Cytometry 46 (2001), 121-135.

[4] J. Baltzer and K.J. Lohe, What's new in prognosis of uterine cancer?, Pathol. Res. Pract. 178 (1984), 635-641.

[5] A. Böcking, C.P. Adler, H.H. Common, M. Hilgarth and B.A. Granzen, Algorithm for a DNA-cytometric diagnosis and grading of malignancy, Analyt. Quant. Cytol. Histol. 6 (1984), $1-8$.

[6] A. Böcking, DNA measurements. When and why?, Compendium on quality assurance, proficency testing and workload limitations in clinical cytology, Tutorials of Cytology, Chicago, Illinois, USA (1995), 170-188.

[7] A. Böcking, F. Giroud and A. Reith, Consensus report of the ESACP task force on standardization of diagnostic DNA image cytometry. European Society for Analytical Cellular Pathology, Analyt. Cell. Pathol. 8 (1995), 67-74.

[8] E. Burghardt, J. Baltzer, A.H. Tulusan and J. Haas, Results of surgical treatment of 1028 cervical cancers studied with volumetry, Cancer 70 (1992), 648-655. 
[9] H.A. Casanova, M. Ohta, T. Kano, Y. Nishida, K. Sakakibara, T. Ishihara and Y. Tomoda, Prognostic significance of nuclear analysis in invasive carcinoma of cervix patients treated by primary radiation, Acta Obst. Gynaec. Jpn. 38 (1986), 243-251.

[10] R. Chatelain, T. Schunck, E.M. Schindler, A.E. Schindler and A. Böcking, Diagnosis of prospective malignancy in koilocytic dysplasias of the cervix with DNA cytometry, J. Reproduc. Med. 34 (1989), 505-510.

[11] R. Chatelain, A. Willms, S. Biesterfeld, W. Auffermann and A. Böcking, Automated Feulgen staining with a temperaturecontrolled machine, Analyt. Quant. Cytol. Histol. 11 (1989), 211-217.

[12] P. Dixon, BMDP Statistical Software, UCLA Press, Department of Biomathematics, University of California, Los Angeles, 1981.

[13] J.E.D. Dyson, C.A.F. Joslin, R.I. Rothwell, P. Quirke, G.G. Khoury and C.C. Bird, Flow cytofluorometric evidence for the differential radioresponsiveness of aneuploid and diploid cervix tumours, Radiotherap. Oncol. 8 (1987), 263272.

[14] F. Giroud, G. Haroske, A. Reith and A. Böcking, 1997 ESACP consensus report on diagnostic DNA image cytometry. Part II: Specific recommendations for quality assurance. European Society for Analytical Cellular Pathology, Analyt. Cell. Pathol. 17 (1998), 201-208.

[15] A. Göppinger, N. Freudenberg, A. Ross, H.G. Hillemans and M. Hilgarth, The prognostic significance of the DNA distribution in squamous-cell carcinomas of the uterine cervix, Analyt. Quant. Cytol. Histol. 8 (1986), 148-151.

[16] G. Haroske, F. Giroud, A. Reith and A. Böcking, 1997 ESACP consensus report on diagnostic DNA image cytometry. Part I: Basic considerations and recommendations for preparation, measurement and interpretion, Analyt. Cell. Pathol. 17 (1998), 189-200.

[17] G. Haroske, J.P.A. Baak, H. Danielsen, F. Giroud, A. Gschwendtner, M. Oberholzer, A. Reith, P. Spieler and A. Böcking, Fourth updated ESACP consensus report on diagnostic DNA image cytometry, Analyt. Cell. Pathol. (2002) (in press).

[18] T. Inoue, T. Chihara and K. Morita, The prognostic significance of the size of the largest nodes in metastatic carcinoma from the uterin cervix, Gynecol. Oncol. 19 (1984), 187-193.

[19] T. Inoue and K. Morita, The prognostic significance of number of positive nodes in cervical carcinoma stages IB, IIA, and IIB, Cancer 65 (1990), 1923-1927.

[20] A. Jakobsen, Ploidy level and short-time prognosis of early cervix cancer, Radiotherapy Oncol. 1 (1984), 271-275.

[21] A. Jakobsen, P. Bichel, G.B. Kristensen and M. Nylands, Prognostic influence of ploidy level and histopathologic differentiation in cervical carcinoma stage Ib, Eur. J. Cancer Clin. Oncol. 24 (1988), 969-972.

[22] T. Kamura, N. Tsukamoto, N. Tsuruchi, T. Saito, T. Matsuyama, K. Akazawa and H. Nakano, Multivariate analysis of the histopathologic prognostic factors of cervical cancer in patients undergoing radical hysterectomy, Cancer 69 (1992), 181-186.

[23] G.B. Kristensen, J. Kaern, V.M. Abeler, B. Hagmar, C.G. Tropé and E.O. Pettersen, No prognostic impact of flow-cytometric measured DNA ploidy and S-phase fraction in cancer of the uterine cervix: a prospective study of 465 patients, Gynecol. Oncol. 57 (1995), 79-85.

[24] C.H. Lai, S. Hsueh, M.Y. Huang, M.F. Chang and Y.K. Soong, The uses and limitations of DNA flow cytometry in stage IB or II cervical carcinoma, Cancer 72 (1993), 3655-3662.

[25] L.C.H.W. Lutgens, B. Schutte, J.M.A. De Jong and F.B.J.M. Thunnissen, DNA content as prognostic factor in cervix carcinoma stage IB-III treated with radiotherapy, $G y$ necol. Oncol. 54 (1994), 275-281.

[26] T. Matsuyama, I. Inoue, N. Tsukamoto, M. Kashimura, T. Kamura, T. Saito and H. Uchino, Stage Ib, IIa, and IIb cervix cancer, postsurgical staging, and prognosis, Cancer 54 (1984), 3072-3077.

[27] G.J. Naus and R.L. Zimmerman, Prognostic value of flow cytometric DNA content analysis in single treatment stage IBIIA squamous cell carcinoma of the cervix, Gynecol. Oncol. 43 (1991), 149-153.

[28] A. Onnis, T. Maggino and M. Marchetti, Surgery as diagnostic and therapeutic moment in the management of cervical cancer, Eur. J. Gynaecol. Oncol. 6 (1985), 20-30.

[29] M.E. Robert and Y.S. Fu, Squamous cell carcinoma of the uterine cervix - a review with emphasis on prognostic factors and unusual variants, Semin. Diagn. Pathol. 7 (1990), 173-189.

[30] D.H. Rutgers, P.M. van der Linden and H.A. van Peperzeel, DNA-flow cytometry of squamous cell carcinomas from the human uterine cervix: The identification of prognostically different subgroups, Radiotherp. Oncol. 7 (1986), 249-258.

[31] L. Sanchez, M. Regh, S. Biesterfeld, R. Chatelain and A. Böcking, Performance of a TV image analysis system as a microdensitometer, Analyt. Quant. Cytol. Histol. 12 (1990), 279-284.

[32] R.E. Scully, T.A. Bonfiglio, R.J. Kurman, S.G. Silverberg and E.J. Wilkinson, Histological Typing of Female Genital Tract Tumours, 2nd edn, Springer-Verlag, Berlin-Heidelberg, 1994.

[33] P. Strang, U. Stendahl, R. Bergström, B. Frankendal and B. Tribukait, Prognostic flow cytometric information in cervical squamous cell carcinoma: a multivariate analysis of 307 patients, Gynecol. Oncol. 43 (1991), 3-8.

[34] B. Tribukait, Nuclear deoxyribonucleic acid determination in patients with prostate carcinomas: clinical research and application, Eur. Urol. 23(Suppl. 2) (1993), 64-76.

[35] R. Willén, A. Himmelmann, E. Långström-Einarsson, M. Fernö, J. Ranstam, B. Baldetorp, J. Skjaerris, J. PrienLarsen, C. Trope and U. Stendahl, Prospective malignancy grading, flow cytometry DNA-measurements and adjuvant chemotherapy for invasive squamous cell carcinoma of the uterine cervix, Anticancer Res. 13 (1993), 1187-1196.

[36] J.M. Yu, H. Zhang, S.Q. Wang, H.Q. Miao, L.H. Yang, Y.T. Chen and G.D. Tian, DNA ploidy analysis of effectiveness of radiation therapy for cervical carcinoma, Cancer 68 (1991), 76-78.

[37] G.M. Zanetta, J.A. Katzmann, G.L. Keeney, W.K. Kinney, S.S. Cha and K.C. Podratz, Flow-cytometric DNA analysis of stages IB and IIA cervical carcinoma, Gynecol. Oncol. 46 (1992), 13-19. 


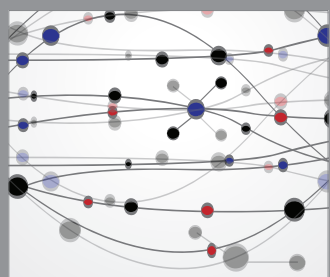

The Scientific World Journal
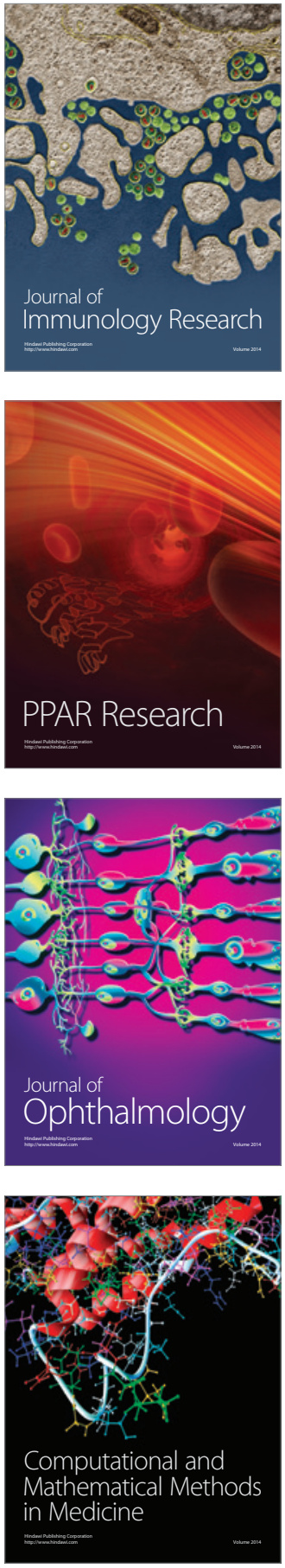

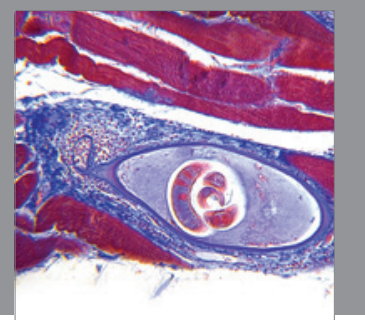

Gastroenterology

Research and Practice
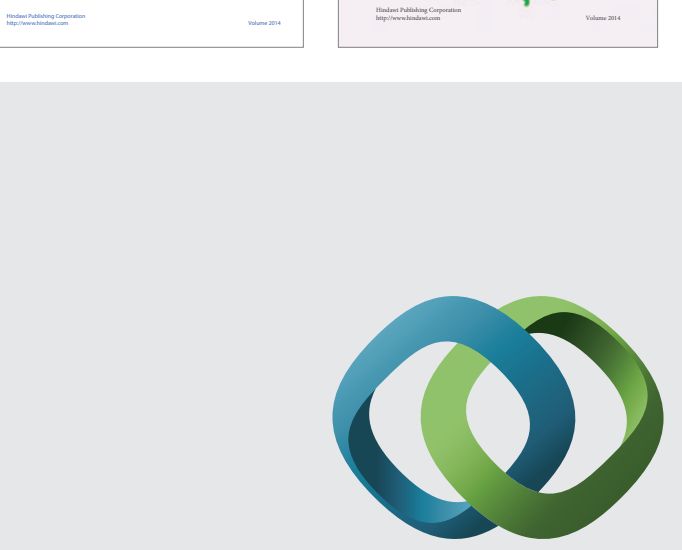

\section{Hindawi}

Submit your manuscripts at

http://www.hindawi.com
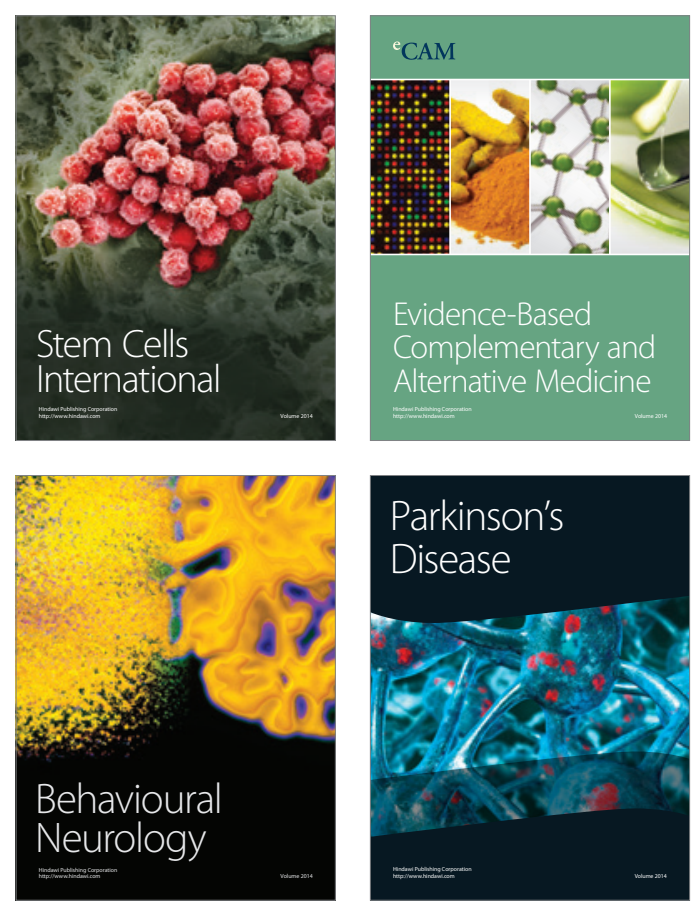

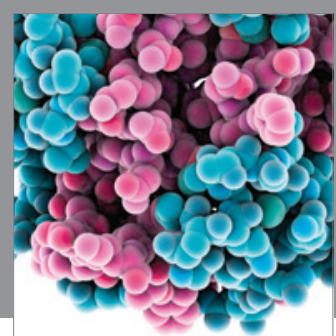

Journal of
Diabetes Research

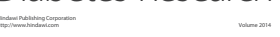

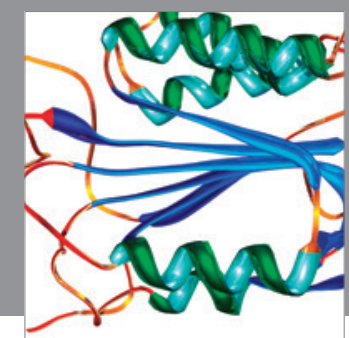

Disease Markers
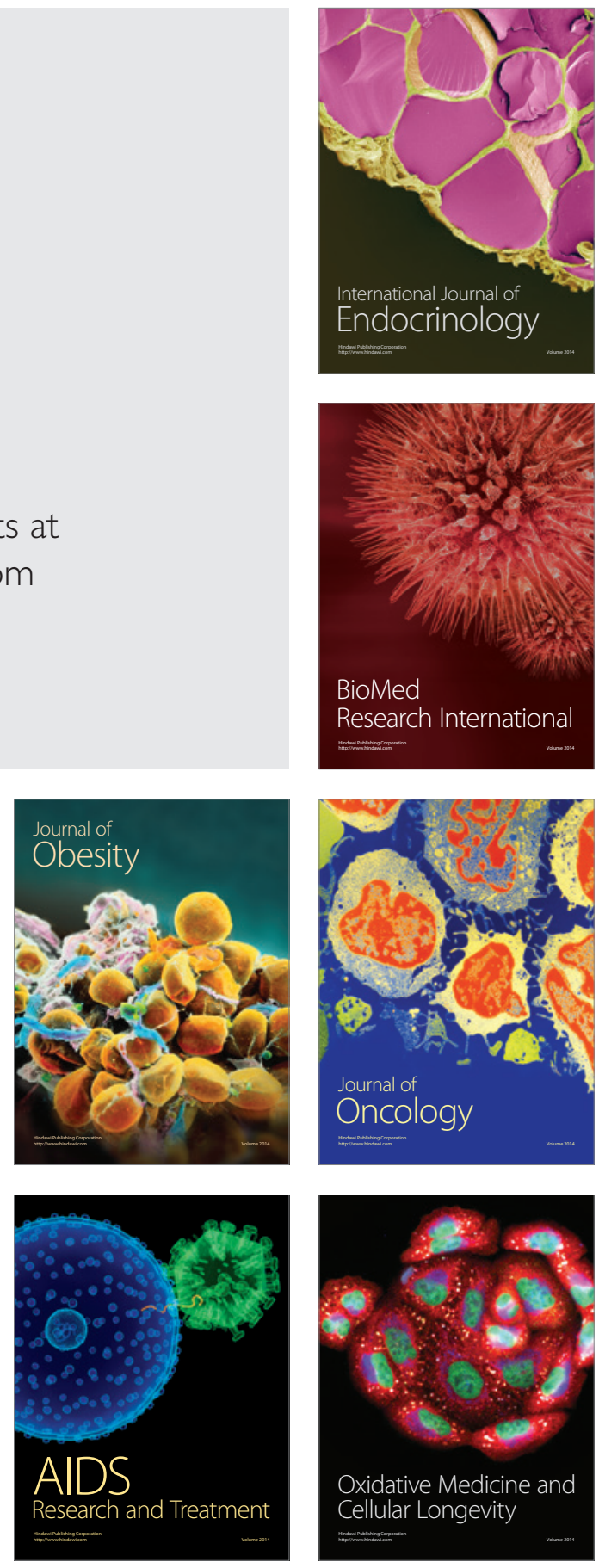